\title{
Erratum to: Explicit Strong Stability Preserving Multistage Two-Derivative Time-Stepping Schemes
}

\author{
Andrew J. Christlieb ${ }^{1}$ - Sigal Gottlieb ${ }^{2}$. \\ Zachary Grant ${ }^{2}$. David C. Seal ${ }^{3}$
}

Published online: 23 March 2016

(C) Springer Science+Business Media New York 2016

\section{Erratum to: J Sci Comput DOI 10.1007/s10915-016-0164-2}

The authors regret that typographical errors appeared in the order conditions, Table 1 in the original publication. These errors included a mistaken factor of 2 on one of the terms in one of the fifth-order conditions, and an omitted equation. The corrected Table 1 of order conditions is provided here.

Table 1 Order conditions for multistage multiderivative methods of the form (6) as in [3]

\begin{tabular}{ll}
\hline$p=1$ & $b^{T} e=1$ \\
$p=2$ & $b^{T} c+\hat{b}^{T} e=\frac{1}{2}$ \\
$p=3$ & $b^{T} c^{2}+2 \hat{b}^{T} c=\frac{1}{3}$ \\
& $b^{T} A c+b^{T} \hat{c}+\hat{b}^{T} c=\frac{1}{6}$ \\
$p=4$ & $b^{T} c^{3}+3 \hat{b}^{T} c^{2}=\frac{1}{4}$ \\
& $b^{T} c A c+b^{T} c \hat{c}+\hat{b}^{T} c^{2}+\hat{b}^{T} A c+\hat{b}^{T} \hat{c}=\frac{1}{8}$ \\
& $b^{T} A c^{2}+2 b^{T} \hat{A} c+\hat{b}^{T} c^{2}=\frac{1}{12}$ \\
& $b^{T} A^{2} c+b^{T} A \hat{c}+b^{T} \hat{A} c+\hat{b}^{T} A c+\hat{b}^{T} \hat{c}=\frac{1}{24}$
\end{tabular}

The online version of the original article can be found under doi:10.1007/s10915-016-0164-2.

Zachary Grant

zgrant@umassd.edu

1 Department of Computational Mathematics Science and Engineering, Department of Electrical Engineering and Department of Mathematics, Michigan State University, East Lansing, MI, USA

2 Department of Mathematics, University of Massachusetts, North Dartmouth, MA, USA

3 Department of Mathematics, U.S. Naval Academy, Annapolis, MD, USA 
Table 1 continued

\begin{tabular}{ll}
\hline$p=5 \quad$ & $b^{T} c^{4}+4 \hat{b}^{T} c^{3}=\frac{1}{5}$ \\
& $b^{T} c^{2} A c+b^{T} c^{2} \hat{c}+\hat{b}^{T} c^{3}+2 \hat{b}^{T} c A c+2 \hat{b}^{T} c \hat{c}=\frac{1}{10}$ \\
& $b^{T} c A c^{2}+2 b^{T} c \hat{A} c+\hat{b}^{T} c^{3}+\hat{b}^{T} A c^{2}+2 \hat{b}^{T} \hat{A} c=\frac{1}{15}$ \\
& $b^{T} c A^{2} c+b^{T} c A \hat{c}+b^{T} c \hat{A} c+\hat{b}^{T} c A c+\hat{b}^{T} c \hat{c}+\hat{b}^{T} A^{2} c+\hat{b}^{T} A \hat{c}+\hat{b}^{T} \hat{A} c=\frac{1}{30}$ \\
& $b^{T}(A c)(A c)+2 b^{T} \hat{c} A c+b^{T} \hat{c}^{2}+2 \hat{b}^{T} c A c+2 \hat{b}^{T} c \hat{c}=\frac{1}{20}$ \\
& $b^{T} A c^{3}+3 b^{T} \hat{A} c^{2}+\hat{b}^{T} c^{3}=\frac{1}{20}$ \\
& $b^{T} A(c A c)+b^{T} A(c \hat{c})+b^{T} \hat{A} c^{2}+b^{T} \hat{A} A c+b^{T} \hat{A} \hat{c}+\hat{b}^{T} c A c+\hat{b}^{T} c \hat{c}=\frac{1}{40}$ \\
& $b^{T} A^{2} c^{2}+2 b^{T} A \hat{A} c+b^{T} \hat{A} c^{2}+\hat{b}^{T} A c^{2}+2 \hat{b}^{T} \hat{A} c=\frac{1}{60}$ \\
& $b^{T} A^{3} c+b^{T} A^{2} \hat{c}+b^{T} A \hat{A} c+b^{T} \hat{A} A c+b^{T} \hat{A} \hat{c}+\hat{b}^{T} A^{2} c+\hat{b}^{T} A \hat{c}+\hat{b}^{T} \hat{A} c=\frac{1}{120}$
\end{tabular}

